\title{
Liquid lens with large focal length tunability fabricating in the polyvinyl chloride/dibutyl phthalate gel tube
}

\author{
Xiaomin Song ${ }^{1,2}$, Dongyang $\mathrm{Li}^{1,2}$, Qingwen $\mathrm{Jin}^{1,2}$, Dagong $\mathrm{Jia}^{1,2}$, Tiegen $\mathrm{Liu}^{1,2}$, Chao \\ Wang $^{3}$, and Hongxia Zhang ${ }^{1,2^{*}}$ \\ ${ }^{1}$ College of Precision Instrument and Optoelectronics Engineering, Tianjin University, \\ Tianjin 300000, China \\ ${ }^{2}$ Key Laboratory of Optoelectronics Information Technical Science, EMC, Tianjin 30000, \\ China \\ ${ }^{3}$ School of Engineering and Digital Arts, University of Kent, Canterbury, Kent,CT2 \\ $7 N T, U K$
}

E-mail: hxzhang@tju.edu.cn

Abstract: Usually, the adaptive liquid lens only has positive focal length, which severely limits the application in imaging and other fields. Therefore, a liquid lens consisting of polyvinyl chloride/dibutyl phthalate gel (PVC/DBP gel), glycerol solution, and glass substrate, is proposed to extend the dynamic focal length range. There is a spherical shape tube formed by PVC/DBP gel under the effect of hydrostatic and the surface tension, which is used to restrict the glycerol solution. The PVC/DBP gel doesn't deform under the effect of an electric field, so the tangent line at the three-phase junction changes with the change of contact angle, which leads to the dynamic focal length range is enlarged. At different voltage values, the proposed lens can be configured to work in three different schemes, namely, converging light, not deflecting light, and diverging light. Here, the proposed lens has a high imaging quality, the resolution is better than $114 \mathrm{lp} / \mathrm{mm}$. The lens with reconfigurable focal length holds great promise in rich application such as in fluorescence detection, beam shaping and adaptive optics.

\section{Introduction}

Fixed optical lenses with a single function of either converging or diverging light severely restricts their applications in fluorescence detection for biochemistry and analytical chemistry ${ }^{1}$, and beam shaping ${ }^{2}$. Adaptive lenses whose focal length can be tuned in either a positive or negative range have been extensively used in biomedical devices ${ }^{3}$, optical free-space communications and tracking ${ }^{4}$, fiber optic networks ${ }^{5}$, aberration compensation ${ }^{6}$, and imaging process ${ }^{7}$.

To obtain a lens system with a focal length that ranges from positive to negative values, an approach that combines a solid lens and an adaptive lens has been developed ${ }^{8-13}$. However such an approach leads to complications in the development of the lens system. Subsequently, certain types of adaptive lenses such as liquid crystal (LC) lens ${ }^{14}$ and liquid lenses ${ }^{15-19}$ have been proposed, which have a dynamic focal length range including positive and negative values. In a typical LC lens, there are three electrodes in the LC cell, and two of them are deposited on both sides of a considerably thin glass substrate. Therefore, it is difficult to manufacture LC lenses because of the complicated structure. Moreover, a characteristic of the LC lens is a tunable refractive index, which can be modulated using the photoelectric effect. Because of the low refractive index change $\delta n$ that can be induced, the dynamic focal length range of a typical LC lens is limited. A liquid lens usually comprises two liquid materials of similar densities but different refractive indices. The key feature of the liquid lens is that it has one tunable liquid-liquid 
surface which can be modulated by electrowetting effect. The common materials used to make adaptive liquid lenses are water drops and nonpolar oil, the contact angle can only vary within the range of $(\pi / 2, \pi)$, which reduces the focal length has decreased to the range of $(21.1 \mathrm{~mm}, 91.9 \mathrm{~mm})^{20}$. The dynamic focal length range of the liquid lens is limited because of contact angle saturation ${ }^{21}$.

To extend the dynamic focal length range limited by the effect of contact angle saturation, and to obtain a lens system with a focal length that can be configured to have both positive and negative values, we report a new approach to design and implement such an adaptive liquid lens. For this purpose, the PVC/DBP gel is reconstructed as an spherical shape, and since the gel undergoes deformation only at a very high voltage ${ }^{22,23}$; therefore, the PVC/DBP gel surface keeps its shape stable under normal operation conditions, so it can be used as a tube to restricted glycerol solution, it is particularly noteworthy that the slope of the tangent decreases nonlinearly. Therefore, the glycerol solution interface can be reconstructed as either a convex wave or a concave wave at different values of DC electric field under the same dynamic contact angle range. Consequently, the focal length can be adjusted from positive to negative values. Compared with the previous lenses normally fabricated in a cylindrical tube, the proposed lens offers a greatly enhanced dynamic focal length range.

\section{Schematic and principle}

Figure 1 shows the front view structure and operating mechanism of the proposed liquid lens. The proposed liquid lens comprises of PVC/DBP gel, glycerol solution, an indium tin oxide (ITO) electrode, and a glass substrate. Because of the stability of PVC/DBP gel under normal operation conditions, the PVC/DBP gel is used as the tube, and the glycerol solution is sealed in the PVC/DBP gel. The proposed lens' surface can be reconfigured with piecewise continuous waves including liquid-PVC/DBP gel and liquid-gas waves; because of the similarity of refractive indices of PVC/DBP gel and glycerol ${ }^{24}$, the liquid-gel surface cannot realize the lens' characteristic, while the liquid-liquid surface has the property to work in the three different schemes, namely, converging light, not deflecting light, and diverging light. In the absence of any electric field, the liquid-gas surface is regard as a convex wave, and the lens has a convex lens-like characteristic with positive focal length, as shown in Fig. 1(a). When a small voltage is applied to the electrode, the contact angle decreases, which leads to the displacement of the initial position of the liquid-liquid surface is displaced. Furthermore, with a higher applied voltage, the liquid-liquid surface gets further deformed from a convex wave to a concave wave, resulting in a negative focal length, as shown in Fig. 1(b).

(a)

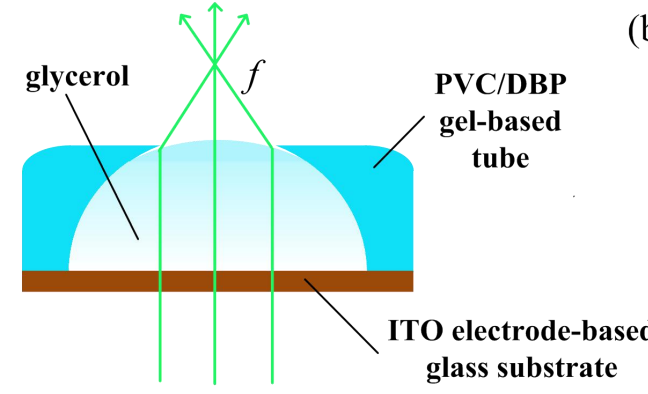

(b)

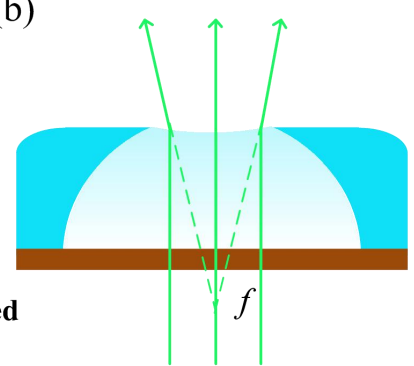

Fig. 1. Front view structure and operation mechanism of the proposed lens. (a) The lens has convex lens-like characteristics. (b) The lens has concave lens-like characteristics. 
To characterize the force condition of the lens, the liquid lens system was established, as shown in Fig.2. In an equilibrium state, there is a balance between surface tension, the applied pressure due to external electrical field, and hydrostatic pressure. Figures 2(a) and (b) show the force condition of the lens, which has convex and concave lens characteristics, respectively.
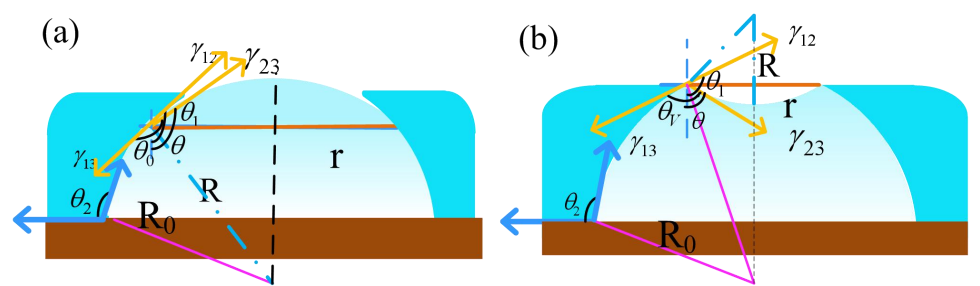

Fig. 2. Analysis of the force balance of variable focus liquid lens. (a) Original equilibrium state when the lens has convex lens-like characteristics. The blue dotted line represents the radius of the liquid-liquid surface (b) A new equilibrium state when the lens has concave lens-like characteristics.

The volume of glycerol $v$ can be determined using the following equation:

$$
v=\frac{2}{3} \pi R^{3}(1-\sin \theta)-\frac{1}{3} \pi r^{2} R \sin \theta+\pi R_{0}^{3} \sin \theta_{1}-\frac{1}{3} \pi R_{0}^{3} \sin ^{3} \theta_{1}+\pi R_{0}^{3} \cos \theta_{0}-\frac{1}{3} \pi r^{3} \cos ^{3} \theta_{0}
$$

where $R, R_{0}, r$ denote the radii of liquid-gel surface, liquid-liquid surface, cross-sectional edge profile of liquid-liquid surface, respectively. $\theta_{1}$ denotes the angle of interfacial tension $\left(\gamma_{12}\right)$ between PVC/DBP gel and glycerol being off Y-direction. Furthermore, angle $\theta$, which is the sum of the contact angle and $\theta_{1}$, denotes the angle of interfacial tension $\left(\gamma_{23}\right)$ between glycerol and gas being off Y-direction. $\theta_{0}$ denotes the contact angle of the droplet of glycerol dropped on the glass substrate with PVC/DBP gel.

According to the relationship between the radius of the liquid-liquid surface, $\mathrm{R}$, and the radius of the cross-sectional edge profile of the liquid-liquid surface, $r$, as shown in Eq. (2).

$$
R=-\frac{r}{\cos \theta}
$$

The total volume of glycerol remains consistent, the total differentiation of $v$ is zero, so

$$
d \theta_{1}=\left[-\frac{2 \sin \theta(1-\cos \theta)}{\cos ^{4} \theta}+\frac{1}{\cos ^{2} \theta}\right] d \theta
$$

In an original equilibrium state, the differentiation of Helmholtz free energy of the lens systems in the absence of any electric field is zero. According to the lens system model, $d F$ can be calculated as follows:

$$
\begin{aligned}
d F & =\gamma_{12} d A_{12}+\gamma_{13} d A_{13}+\gamma_{23} d A_{23} \\
& =\left(\gamma_{12}-\gamma_{13}\right) \cdot 2 \pi r^{2} \cos \theta_{1} d \theta_{1}+\gamma_{23}\left[4 \pi R(1-\sin \theta) d R-2 \pi R^{2} \cos \theta d \theta\right]
\end{aligned}
$$

where $w$ denotes the interfacial energy. $A_{12}, A_{13}, A_{23}$ denote the contact areas between PVC/DBP gel and glycerol, between PVC/DBP gel and gas, and between glycerol and gas, respectively. $\gamma_{12}, \gamma_{13}$ and $\gamma_{23}$ are the interfacial tensions between PVC/DBP gel and glycerol, between PVC/DBP gel and gas, and between glycerol and gas, respectively. Because the total differentiation of Helmholtz free energy is zero, Applying Eq. (2) to Eq. (4), 


$$
d \theta_{1}=\frac{\gamma_{23}}{\gamma_{12}-\gamma_{13}} \cdot \frac{\left[4 \pi R(1-\sin \theta) d R-2 \pi R^{2} \cos \theta d \theta\right]}{2 \pi r^{2} \cos \theta_{1}}
$$

According to Eq. (4) and (5), at the absence of the electric field, the Young's equation in the lens system can be expressed as follows:

$$
\cos \theta_{0}=\frac{\gamma_{13}-\gamma_{12}}{\gamma_{23}}
$$

When voltage is applied to the electrode, the dynamic contact angle $\cos \theta_{v}$ can be calculated as follows:

$$
\cos \theta_{v}=\cos \theta_{0}+\frac{\varepsilon}{2 \gamma_{12} d_{0}} U^{2}
$$

In Eq. (7), $\varepsilon$ denotes the relative permittivity of the dielectric layer, $U$ denotes the potential difference applied, and $d_{0}$ denotes the dielectric thickness.

Similar to a solid lens, the focal length $(f)$ of the performed lens can also be expressed as follows, but the parameter $R$ depends on the dynamic contact angle.

$$
f=\frac{R}{n-1}=-\frac{r}{\cos \theta(n-1)}=-\frac{R}{\left(\cos \theta_{0}+\frac{\varepsilon_{0} \varepsilon_{r}}{2 \gamma_{12} d_{0}} U^{2}\right)(n-1)}
$$

To demonstrate the superior performance of our proposed liquid lens design, we also analyze a typical liquid lens restricted in a cylindrical tube those focal length can be approximated as Eq.(8). However, as shown in Fig. 2, because of the relationship between the radius of the liquid-liquid interface and the radius of the PVC/DBP gel tube, the focal length can be calculated as follows,

$$
\begin{aligned}
f & =-\frac{R_{0} \cos \theta_{1}}{\cos \left(\theta_{v}-\pi+\theta_{1}\right)(n-1)} \\
& =-\frac{R_{0} \cos \theta_{1}}{\left[-\left(\cos \theta_{c}+\frac{\varepsilon_{0} \varepsilon_{r}}{2 \gamma_{12} d_{0}} U^{2}\right) \cos \theta_{1}+\sqrt{1-\left(\cos \theta_{c}+\frac{\varepsilon_{0} \varepsilon_{r}}{2 \gamma_{12} d_{0}} U^{2}\right)^{2}} \sin \theta_{1}\right](n-1)}
\end{aligned}
$$

where $n$ denotes the refractive index of glycerol. Because of the liquid-liquid interface can range from being a convex wave to a concave wave, $\mathrm{R}$ can be as positive or negative accordingly.

From Eq. (8), it demonstrated the focal length of the lens fabricated in a cylindrical tube only depends on the contact angle; usually, the contact angle is in the range $(\pi / 2, \pi)$, which results in the focal length in a positive range. However, for our lens, it depends not only on the contact angle, but also on the slop of tangent line at the contact point; the slop of tangent line is expressed by the parameter $\theta_{1}-\pi \quad\left(0<\theta_{1}-\pi<\pi / 2\right)$, as shown in Eq. (9). Hence, the lens has not only the positive focal length, but also the negative values by tuning the applied voltage.

\section{Experiments}

Here, we prepared PVC/DBP gel by thawing a dissolved mixture of $3 \mathrm{~g}$ PVC, $30 \mathrm{ml}$ THF, and $36 \mathrm{ml} \mathrm{DBP}$ at $65{ }^{\circ} \mathrm{C}$ for 5 hours. Then, we obtained a uniform solution and the refractive index of the PVC/DBP gels was measured to be $\sim 1.49$ [20]. Glycerol (1 ml) was dropped onto the glass substrate, and a glycerol droplet with a diameter of $\sim 7 \mathrm{~mm}$ 
was formed. Subsequently, we slowly dropped the PVC/DBP gel into the quartet container. Note that glycerol was not fully immersed, and the section that is not immersed could act as a lens whose optical aperture was $\sim 7 \mathrm{~mm}$ in the absence of any DC voltage.

To characterize the focusing property of the fabricated lens, an experimental setup shown in Fig.3 was built. At the initial equilibrium state (no DC voltage applied), we adjusted the microscope objective to bring the object into the focus state, such that we observed the object's clear image on a charge-coupled device (CCD) camera. Then, we turned on the voltage source and gradually increased it. We obtained the images using the CCD camera at different voltage values.

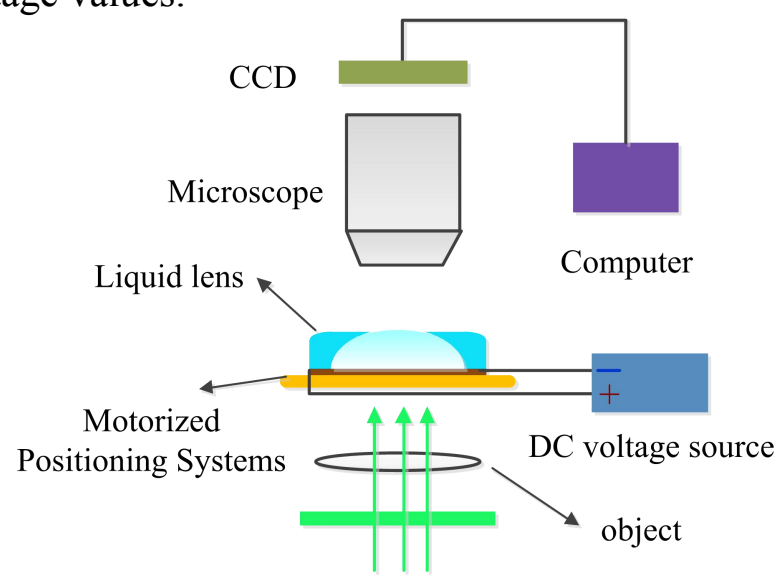

Fig. 3. Experimental setup for measuring the focusing property of the liquid lens at various voltages

\section{Results and Analysis}

A thin glass plate with two black horizontal bars on the top of it was selected as an object and placed in the optical microscope system as shown in Fig. 3. The prepared liquid lens was placed $2 \mathrm{~mm}$ away from the object. At the voltage-off state, a clear image of the object was displayed on the CCD camera, as shown in Fig. 4(a). Subsequently, with the increase of the applied voltage, the corresponding images obtained using the CCD became blurry. Fig. 4(b) shows the image obtained at $\mathrm{V}=60 \mathrm{~V}$. As the applied voltage reached $180 \mathrm{~V}$, a blurrier image was obtained using the CCD camera, as shown in Fig. $4(\mathrm{c})$. Furthermore, as the applied voltage reached $280 \mathrm{~V}$, the object "=" could be barely recognized, as shown in Fig. 4(d).

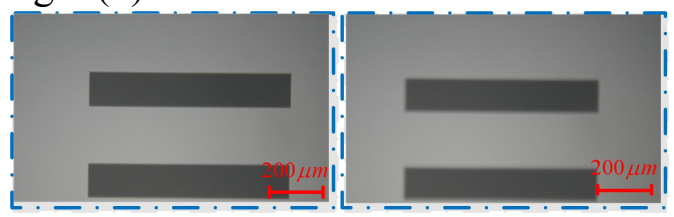

(a)

(b)

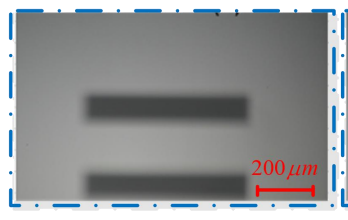

(c)

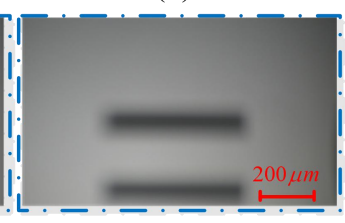

(d)

Fig.4. The image of object " $="$ in the defocused state at different voltage values.(a) $V=0 \mathrm{~V}$, (b) $V=60 \mathrm{~V}$, (c) $V=180 \mathrm{~V}$, (d) $V=280 \mathrm{~V}$. 
These results indicate that the lens has a dynamic focal length range when an electric field is applied. To study the light-focusing property of the proposed lens, we placed the lens under an optical microscope. Let the distance from the proposed lens to a beam profiler be denoted by $L$. Fig. 5(a)shows the laser beam intensity profile observed at $V=100 \mathrm{~V}$ and $L=5 \mathrm{~mm}$. The intensity of the peaks was observed to reach as high as $\sim 85$ arbitrary units, as shown in Fig. 5(b). Moreover, the intensity distribution under $L=15 \mathrm{~mm}$ is shown in Fig. 5(c), and the corresponding intensity of the peaks is $\sim 90$ arbitrary units, as shown in Fig. 5(d). This revealed that while the intensity increases, the spot size decreased, thereby indicating convex lens-like characteristics of the lens at $V=100 \mathrm{~V}$. Figures $5(\mathrm{e})$ and $(\mathrm{g})$ show the intensity distribution at $V=185 \mathrm{~V}, L=5 \mathrm{~mm}$ and at $V=$ $185 \mathrm{~V}, L=15 \mathrm{~mm}$, respectively. The change of the spot size can be neglected. The intensity of the peaks was observed to be more than 80 and 75 arbitrary units, respectively, as validated from Figs. 5(f) and (h). Such results revealed that the lens did not deflect light. In a third experiment, the intensity distribution was detected by the beam profiler when the applied voltage $V=280 \mathrm{~V}$. Figures $5(\mathrm{I})$ and (k) show the intensity distribution at $L=5 \mathrm{~mm}$ and $L=15 \mathrm{~mm}$, respectively, and the intensity of the peaks was observed to be $\sim 80$ and 75 arbitrary units, respectively, as shown in Fig.5 (J) and (L). Because the spot size was larger than the size of the beam profiler, we adjusted the detection threshold to 35 arbitrary units. In this situation, the radius of the spot was observed to increase when L is enlarged. For these reasons, it can be inferred that the lens had concave lens-like characteristics.

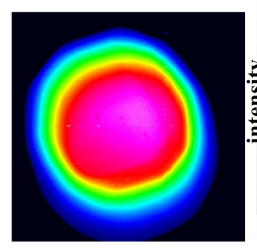

(a)

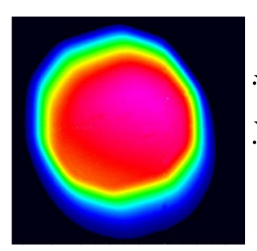

(e)

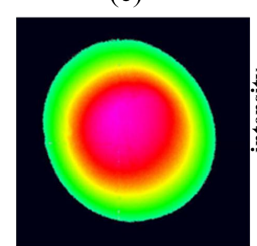

(I)

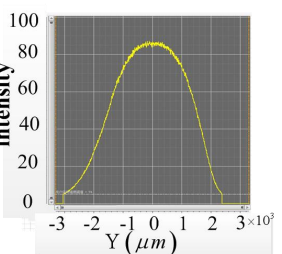

(b)

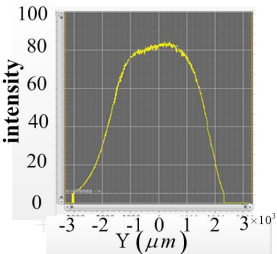

(f)

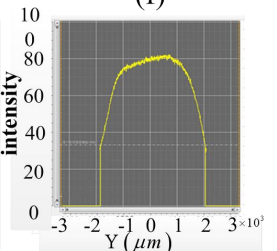

(J)

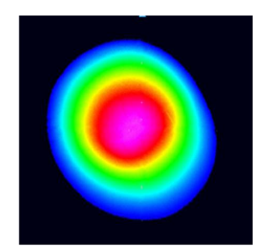

(c)

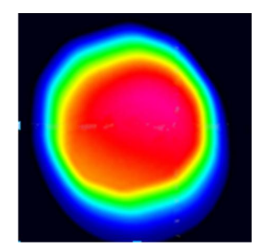

(g)

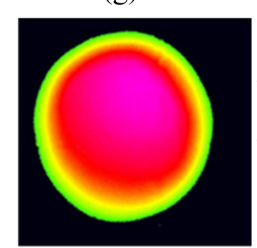

$(\mathrm{K})$

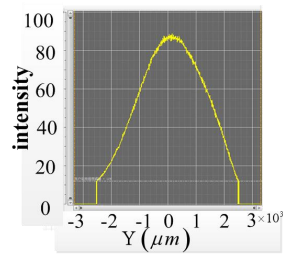

(d)

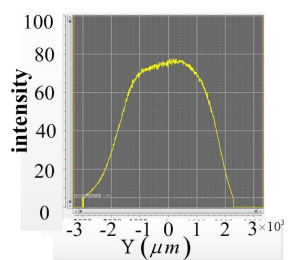

(h)

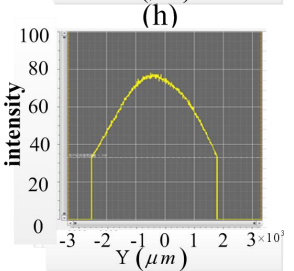

(L)

Fig.5. At different voltage values, the lens has three optical properties, namely, converging light, not deflecting light, and diverging light, respectively. L denotes the distance between the lens and the beam profiler. (a), (e), and (I) are obtained at $\mathrm{L}=5 \mathrm{~mm}$. (c), (g), and (k) are obtained at $\mathrm{L}=15 \mathrm{~mm}$. (a) and (c) indicate that light through the lens is convergent at $\mathrm{V}=100 \mathrm{~V}$. (b) and (d) are the corresponding intensity in the Y-direction. (e) and (g) indicate that light through the lens is not deflected at $\mathrm{V}=185 \mathrm{~V}$. (f) and (h) are the corresponding intensity values in the Y-direction. (i) and $(\mathrm{k})$ indicate that light through the lens is divergent at $\mathrm{V}=280 \mathrm{~V}$. (J) and (L) are the corresponding intensity values in the Y-direction. 
To quantitatively measure the focal length of the proposed lens, we adjusted the objective of the microscope to bring the object into the focused state at various voltage values, and we detected clear images using the CCD. Compared with the original image, the amplification factor $(\beta)$ can be calculated by comparing with the original image. The focal length of the proposed lens at different voltages can be calculated on the basis of the relationship between the amplification factor $(\beta)$ and focal length $(f)$, as shown in Eq. (16).

$$
f_{x}=\frac{\beta l}{\beta-1}
$$

At the voltage-off state, the focal length of the proposed lens composed of PVC/DBP gel and glycerol was calculated to be $20.87 \mathrm{~mm}$. When a voltage was applied, the focal length increases slowly until the applied voltage reached $80 \mathrm{~V}$. Then, the focal length was observed to increase sharply; however, the focal length became negative at $V=200 \mathrm{~V}$, i.e., $-188.47 \mathrm{~mm}$. On increasing the applied voltage, the focal length increased sharply again, but the focal length growth slows down after the applied voltage became $>240$ V. At $V=$ $280 \mathrm{~V}$, the focal length was detected to be $-22.57 \mathrm{~mm}$, as shown in Fig.6.

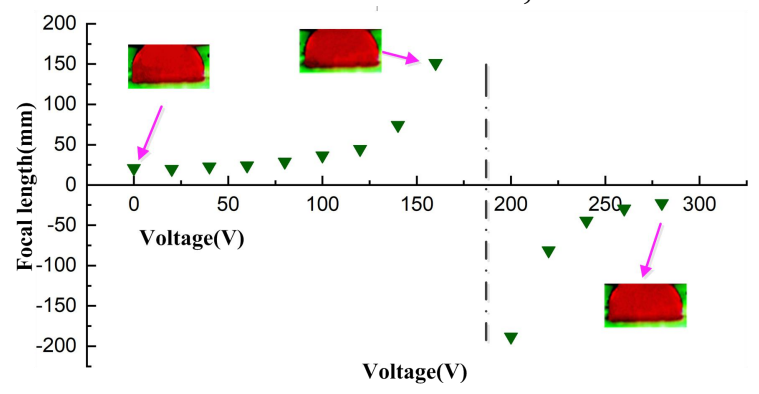

Fig.6. Focal length of the lens is dependent on the applied voltage.

Here, the liquid-glycerol surface was measured using an optical microscope. The insets in Fig. 6 also shows the liquid-glycerol surface's profile at $V=0 \mathrm{~V}, V=160 \mathrm{~V}$, and $V=280$ $\mathrm{V}$, which indicates that the liquid-gel surface is not deformed when an electric field is applied. Therefore, the liquid-liquid surface has the lens characteristic.

The imaging performance of the proposed liquid lens is explored with a USAF1951 resolution target as the object. In the absence of a DC electric field, a clear image of the resolution target was obtained using the CCD camera. It could resolve up to element 6 in group 6, and the image resolution was observed to be $\sim 114 \mathrm{lp} / \mathrm{mm}$, as shown in Fig. 7(a). On increasing the applied voltage from $V=120 \mathrm{~V}$ to $V=280 \mathrm{~V}$, the corresponding clear images were detected using the CCD camera, as shown in Fig. 7(c), (e), and (g), and the image resolution was also observed to be up to $114 \mathrm{lp} / \mathrm{mm}$.

Furthermore, a circular letter with a radius $\sim 3.5 \mathrm{~mm}$ was placed under the proposed lens as an object. We adjusted the objective of the microscope to bring the object in the focused state, and clear images were obtained by CCD camera at $V=0 \mathrm{~V}, V=120 \mathrm{~V}, V=$ $185 \mathrm{~V}$, and $V=280 \mathrm{~V}$, as shown in Fig. 7 (b), (d), (f), and (h), respectively. The texture of the object clearly emerged in the images, and the edge information was distinguishable. Such results imply the lens has high imaging quality, which is irrelevant to the applied voltage. 


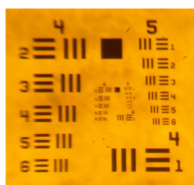

(a)

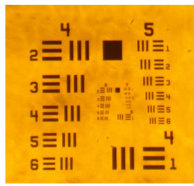

(e)

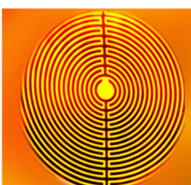

(b)

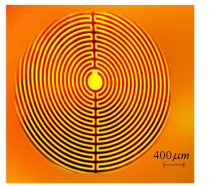

(f)

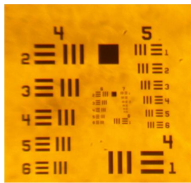

(c)

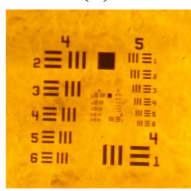

(g)

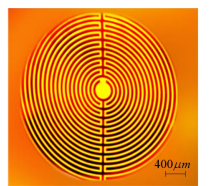

(d)

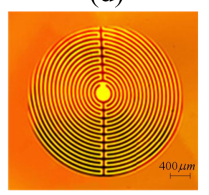

(h)

Fig. 7. Image of the resolution target observed through the single liquid lens. (a)-(d) Images in the defocused state.In (a)-(d), the driven voltage is $V=0 \mathrm{~V}, V=185 \mathrm{~V}, V=280 \mathrm{~V}$, and $V=280 \mathrm{~V}$, respectively. (d)-(f) Images in the focused state. In (e)-(h), the driven voltage is corresponding to that in (a)-(d).

To characterize the response of the proposed lens, A collimate beam was passed through the axis of the proposed lens. A Si amplified detector (Thorlabs PDA10A-EC) was placed after the lens, and the resulting electrical signal was recorded using an oscilloscope. The experimental response of the lens is characterized in Fig. 8. The response time of the lens is about $100 \mathrm{~ms}$ for generated wave from a very short rise time input exponential voltage, as shown in Fig. 8 (a). For a longer input rise time of $600 \mathrm{~ms}$, the proposed lens interface follows the input voltage shape, as shown in Fig. 8 (b).

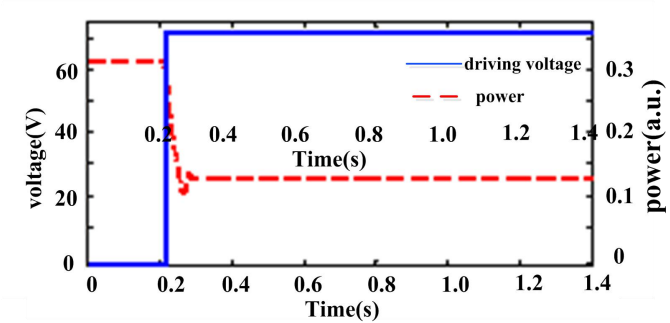

(a)

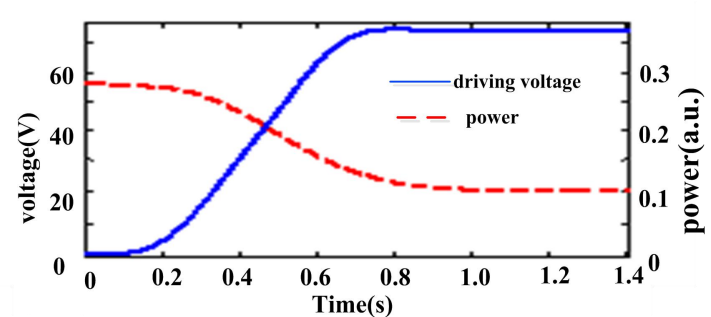

(b)

Fig.8 experimental response characteristics for different input exponential rise times (a) the input time is $1 \mathrm{~ms}$,(b) the input time is $600 \mathrm{~ms}$.

\section{Discussions}

In this study, the PVC/DBP gel-based tube is formed under the effect of interfacial tension difference, which effectively simplifies the production process of the lens. Because of the similarity of the refractive indices of PVC/DBP gel and glycerol solution, the liquid-gel surface doesn't have the lens characteristic, but the spherical gel-based tube makes the tangent being off Y-direction, and the offset degree increases with the increase of the applied voltage. Usually, the contact angle is in the range of $(\pi / 2, \pi)$, which leads to the focal length ranges in positive values. However, because of the offset tangent, the proposed lens can be configured to work in three different schemes, namely, converging light, not deflecting light, and diverging light.

Here, $60 \mu l$ glycerol was dropped onto the glass substrate, subsequently, the PVC/DBP gel was added into the rectangular chamber, and the lens with $7 \mathrm{~mm}$ optical aperture has been implemented. The focal length depends on the applied voltage and the deviated angle of the tangent line being off Y-direction, which decreases with the deviated angle, but increases with the applied voltage. Therefore, even though the contact angle changes within $(\pi / 2, \pi)$, the focal length can be tuned to a negative value, which can change in the range $(-\infty,-22.57 \mathrm{~mm}) \cup(20.87 \mathrm{~mm},+\infty)$. 
When the applied voltage changes in a small range, according to the approximation theory, there is a linear relationship between $1 / f$ and $U^{2}$. Because of the change of slant angle of the tube at three-phase junction, the slope changes at different voltage tuning ranges, as shown in Fig. 9.

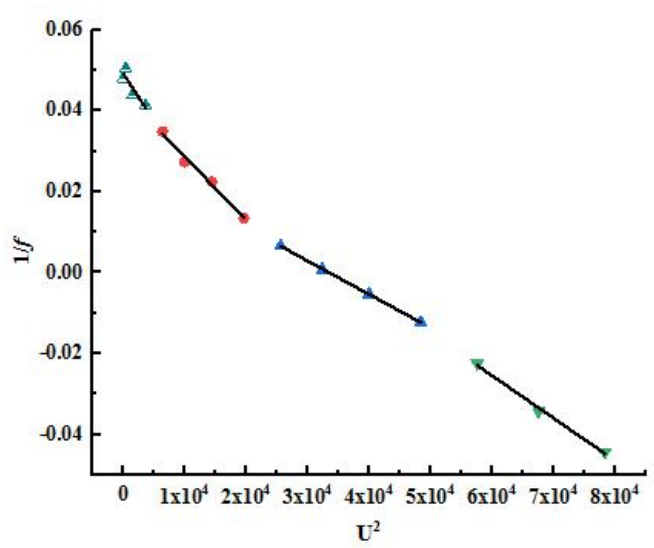

Fig.9 The relationship between $1 / f$ and $\mathrm{U}^{2}$

As a result of the similar refractive index of glycerol and gel, only the liquid-gas surface has the lens characteristic, which can be seen as the center part of the glycerol droplet profile, and the width of liquid-liquid surface is far less than the diameter of the glycerol droplet, therefore, so the imaging performance (USAF 1951) will improve significantly. The image resolution was also observed to be up to $114 \mathrm{lp} / \mathrm{mm}$. Aberration is the main factor that affects the automatic focal length measurement system ${ }^{25}$; hence, the method of measuring focal length with high imaging quality is reasonable.

Furthermore, the liquid-gas surface depends on the profile of the glycerol droplet, whose radius of curvature increases with the diameter of the glycerol droplet, which leads to the improvement of focusing ability. The threshold voltage is lower as the diameter of the glycerol droplet decreases, and the focusing ability will also promote as the diameter of the glycerol droplet decrease. The threshold voltage of our lens is smaller than the normal adaptive lens with two liquids, and it will decreases with the decrease of the glycerol droplet.

\section{Conclusion}

In this study, a reconfigurable liquid lens was designed and demonstrated, there are two tubes reconstructed by glass substrate and PVC/DBP gel, respectively. The PVC/DBP gel was configured with spherical wave, and the tube with PVC/DBP gel and glycerol solution was designed and demonstrated. The lens could be configured as either convex or concave with the application of different voltage values. Because of the deformation of the PVC/DBP gel, the focal length has been extended which is limited by the contact angle saturation. At the voltage-off state, the lens was observed to act as converging with the focal length being $20.87 \mathrm{~mm}$. On increasing the applied voltage, the focal length was observed to tend to $\infty$ when the applied voltage reached $185 \mathrm{~V}$. Then, the lens was proved to have concave lens-like characteristics. When the applied voltage gradually increases to $280 \mathrm{~V}$, the focal length changes from $-\infty$ to $-22.57 \mathrm{~mm}$. Moreover, the lens has high imaging quality.

\section{Acknowledgments}

The work was supported by National Natural Science Foundation of China (NSFC) 


\section{$(61675153,61811530334)$.}

\section{References}

(1) Ju Ha Kim, Jun Geun Shin, Eun Seo Choi, and Tae Joong Eom, "Intraoperative OCT for surgical microscope with sensitivity drop and depth of focus correction based on variable focus and dynamic reference," Opt. Express 2019,27: 3448-3459

(2) Juliet T. Gopinath, Victor M. Bright, Carol C. Cogswell, et al, "Simulation of electrowetting lens and prism arrays for wavefront compensation," Appl. Opt. 2012,51:6618-6623.

(3) Syed Azer Reza, Tariq Shamim Khwaja, Hamid Suleman, "A dual-mode variable optical power splitter using a digital spatial light modulator and a variable focus lens," Opt. Fiber Technol. 2019,47: $118-125$.

(4) J. Knittel, H. Richter, M. Hain, S. Somalingam, T. Tschudi, "Liquid crystal lens for spherical aberration compensation in a blu-ray disc system, "IEEE Proc. , Sci. Meas. Technol. 2005, $152: 15-18$

(5) J.M.Jabbour, M.A. Saldua, J. N. Bixler, and K. C. Maitlans, "Confocal endomicroscopy: instrumentation and medical applications, " Ann. Biomed. Eng. 2012,40, 378-397.

(6) Chaolong Song, Nam-Trung Nguyen, Yit Fatt Yap, Trung-Dung Luong, Anand Krishna Asundi. "Multi-functional, optofluidic, in-plans, bi-concave lens: tuning light beam from focused to divergent,"Microflu. Nanoflu. 2011,10:671-678.

(7) Mert Serkan, and Hulya Kirkici. "Optical beam-shaping design based on aspherical lenses for circularization, collimation, and expansion of spherical laser beams," Appl.Optics, 2008,47:230-241.

(8) Yi-Hsin Lin, Ming-Syuan Chen, and Hung-Chun Lin. "An electrically tunable optical zoom system using two composite liquid crystal lenses with a large zoom ratio," Opt. Express 2011,19:4714-4721.

(9) Yi-Hsin Lin, Hongwen Ren, Kuan-Hsu Fan-Chiang, and Shin-Tson Wu. "Tunable-focal cylindrical liquid crystal lens," Japanese Journal of App. Physics 2005,44: 243-244.

(10) Yun-Hsing Fan, Hongwen Ren, Xiao Liang, Haiying Wang, and Shin-Tson Wu. "Liquid crystal microlens arrays with switchable positive and negative focal lengths," J. Disp. Technol. 2005,1:151-156.

(11) Hongwen Ren, Shin-Tson Wu. "Adaptive liquid crystal lens with large focal length tunability," Opt. Express 2006,14: 11292-11298.

(12) Ziqian He, Yunhan Lee, Debashis Chanda, and Shin Tson. "Adaptive liquid crystal microlens array enabled by two-photon polymerization," Opt. Express 2018,26:21184-21193.

(13) Ziqian He, Yunhan Lee, Ran Chen, Debashis Chanda, and Shin Tson. "Switchable pancharatnam-berry microlens array with nano-imprinted liquid crystal alignment, ” Opt. Lett. 2018, 43:5062-5065.

(14) Bin Wang, Mao Ye, and Susumu Sato. "Liquid crystal lens with focal length variable from negative to positive values," IEEE Photonics Technology Letters 2006,18:79-81.

(15) Liang Dong, Abhishek K. Agarwal, David J. Beebe, and Hongrui Jiang. "Adaptive liquid microlenses activated by stimuli-responsive hydrogels," Nature ,2006,442:551-554.

(16) $\mathrm{Su} \mathrm{Xu,} \mathrm{Yifan} \mathrm{Liu,} \mathrm{Hongwen} \mathrm{Ren,} \mathrm{and} \mathrm{Shin-Tson} \mathrm{Wu.} \mathrm{"A} \mathrm{novel} \mathrm{adaptive} \mathrm{mechanical-wetting} \mathrm{lens} \mathrm{for}$ visible and near infrared imaging," Opt. Express 2010,18(12):12430-12435.

(17) Ryoichi Kuwano, Tsuyoshi Tokunaga, Yukitoshi Otani, and NOrihiro Umeda. "Liquid pressure varifocus lens," Opt. Review 2005,12(5): 405-408.

(18) WeiYang Lim, Omkar D. Supekar, Mo Zohrabi, Juliet T. Gopinath, and Victor M. Bright. "Liquid combination with high refractive index contrast and fast scanning speeds for electrowetting adaptive optics," Langmuir,2018,34: 14511-14518.

(19) J. C. Reis, I. M. S. Lampreia, A. F. S. Santos, M. L. C. J. Moita, and G. Douhéret, "Refractive index of liquid mixtures: theory and experiment," Chem. Phys. Chem. 2010,11:3722-3733.

(20) Lei Li, Chao Liu, Hongwen Ren, Huan Deng, and Qiong-Hua Wang. "Annular folded electrowetting liquid lens, " Opt. Lett. 2015,20: 1968-1971.

(21) Xiangming Li, Hongmiao Tian, Jinyou Shao, Yucheng Ding, Xiaoliang Chen, Li Wang, Bingheng Lu, "Decreasing the saturated contact angle in electrowetting-on-dielectrics by controlling the charge trapping at liquid-solid interfaces", Adv. Funct. Mater. 2016,26: 2994- 3002.

(22) Sang-Youn Kim, Myoung Yeo, Eun-Jae Shin, Won-Hyeong Park, Jong-Seok Jang, Byeong-Uk Nam, and Jin Woo Bae. "Fabrication and evaluation of variable focus and large deformation plano-convex 
microlens based on non-ionic poly(vinyl chloride) /dibutyl adipate gels," Smart Mater. Struct. 2015,24: 115006.

(23) Hong Xia, Midori Takasaki, and Toshihiro Hirai. "Actuation mechanism of plasticized PVC by electric field," Sensor. Actuat. A-Phys. 2019,157(2): 307-312.

(24) J.C. Reis, I. M. S. Lampreia, A. F. S. Santos, M. L. C. J. Moita, and G. Dohéret, "Refractive index of liquid mixures: theory and experiment," Chem. Phys. Chem. 2010,11(17):3722-3733.

(25) Daniel R. Neal R. James Copland, David A. Neal, Daniel M. Topa, Phillip Riera, "Measurement of lens focal length using multicurvature of Shack_Hartmann wavefront data," Proc. SPIE 2004,5523. 

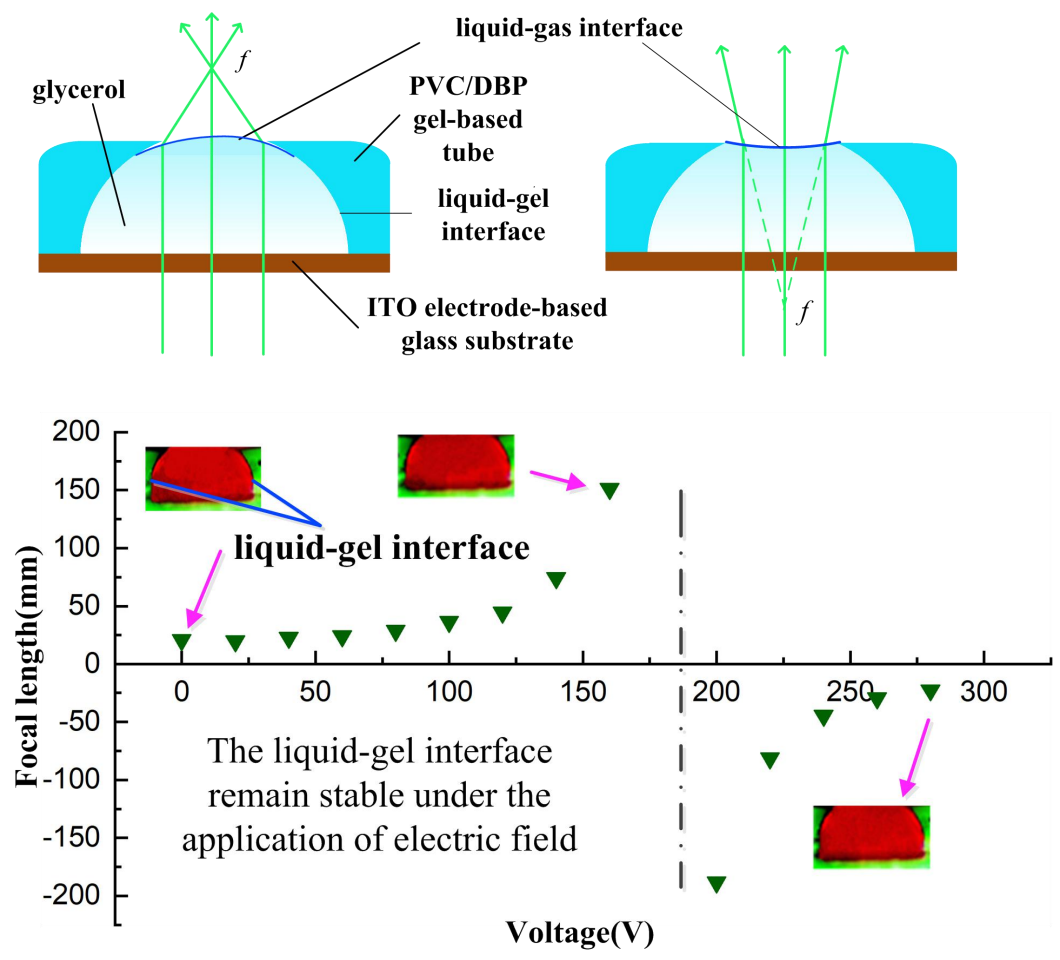